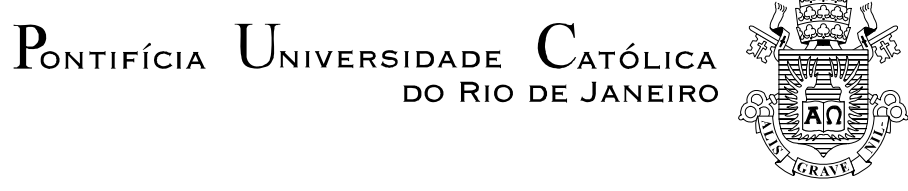

Renata de Oliveira Razuk

Do Inglês L1 ao Português L3 Passando pelo Espanhol L2:

Transferências em Regência/Transitividade Verbal, com

Foco nas Preposições

Tese de Doutorado

Tese apresentada ao Programa de Pós-graduação em Letras do Departamento de Letras da PUC-Rio como parte dos requisitos parciais para obtenção do título de Doutor em Letras.

Orientador(a): Profa. Dra. Eneida do Rêgo Monteiro Bomfim Coorientador(a): Profa. Dra. Rosa Marina de Brito Meyer

Rio de Janeiro

Agosto de 2008 
Renata de Oliveira Razuk

\section{Do Inglês L1 ao Português L3 Passando pelo Espanhol L2: Transferências em Regência/Transitividade Verbal, com Foco nas Preposições}

Tese apresentada como requisito parcial para obtenção do grau de Doutor pelo programa de Pós-Graduação em Letras do Departamento de Letras do Centro de Teologia e Ciências Humanas da PUC-Rio. Aprovada pela Comissão Examinadora abaixo assinada.

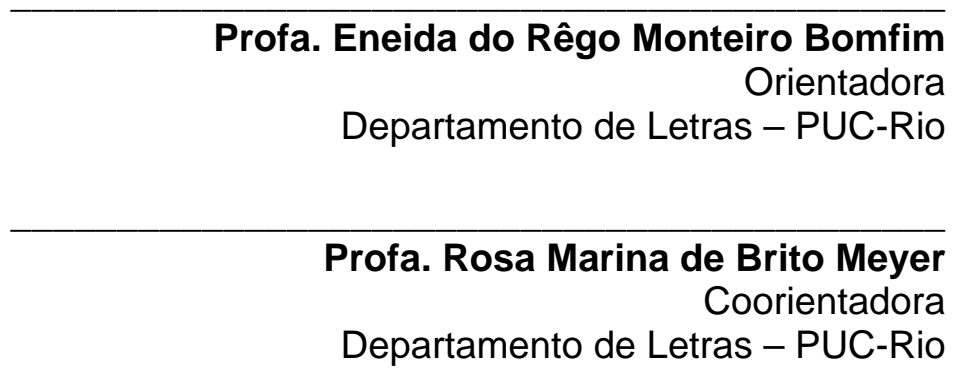

Profa. Adriana Ferreira de Souza Albuquerque

Departamento de Letras - PUC-Rio
Profa. Patricia Maria Campos de Almeida
Faculdade de Letras - UFRJ

Prof. Ricardo Borges Alencar

Departamento de Letras - PUC-Rio

Profa. Matilde Virginia Ricardi Scaramucci

Universidade de Campinas - UNICAMP

Prof. Paulo Fernando Carneiro de Andrade

Coordenador Setorial do Centro de Teologia e Ciências Humanas - PUC-Rio

Rio de Janeiro, de de 
Todos os direitos reservados. É proibida a reprodução total ou parcial do trabalho sem autorização da universidade, da autora e das orientadoras (orientadora e coorientadora).

\section{Renata de Oliveira Razuk}

Graduou-se em Comunicação Social, Jornalismo, na Uerj (Universidade do Estado do Rio de Janeiro) em 1996 e obteve o título de Mestre em Administração Social, Marketing, pelo Coppead/UFRJ (Universidade Federal do Rio de Janeiro) em 2001. Desde então, trabalha (concursada) no Tribunal de Contas do Estado do Rio de Janeiro, onde tem atuado, concomitantemente, como redatora e revisora na Coordenadoria Setorial de Atas, Publicações e Taquigrafia - CST e como membro da equipe editorial na Comissão Permanente de Estudos e Pesquisas - Copep/COE. Concluiu o curso de especialização em Formação de Professores de Português para Estrangeiros da PUC-Rio em 2002, a partir de quando vem se interessando por ensino e pesquisa em língua portuguesa voltada para falantes não-nativos - especialmente o português como terceira língua (PL3) -, tendo publicado alguns artigos e participado de diversos encontros na área, incluindo o recente Congresso Internacional da Aila (Essen, 2008), cujo tema principal foi o Multilinguismo ("Multilingualism: challenges \& opportunities").

Ficha Catalográfica

Razuk, Renata de Oliveira

Do Inglês L1 ao Português L3 passando pelo Espanhol L2: transferências em regência/transitividade verbal, com foco nas preposições / Renata de Oliveira Razuk ; orientadora: Eneida do Rêgo Monteiro Bomfim; coorientador: Rosa Marina de Brito Meyer. -2008 .

334 f. ; $30 \mathrm{~cm}$

Tese (Doutorado em Letras) - Pontifícia Universidade Católica do Rio de Janeiro, Rio de Janeiro, 2008.

Inclui bibliografia

1. Letras - Teses. 2. Aquisição de terceira língua (AL3). 3. Línguas em contato. 4. Ensino de inglês como língua estrangeira. 5. InglêsL1-espanholL2-portuguêsL3. 6. Análise de transferências. 7. Regência/transitividade verbal. 8. Gramática de valências. 9. Relativismo linguístico. 10. Preposições. I. Bomfim, Eneida do Rêgo Monteiro. II. Meyer, Rosa Marina de Brito. III. Pontifícia Universidade Católica do Rio de Janeiro. Departamento de Letras. IV. Título. 
Dedico esta grande conquista aos meus maiores amores: aos meus pais, Foze e Heloisa Razuk, pela vida; ao meu irmão, Ricardo Razuk, pela alegria de viver; ao meu marido, Eliésio S. Júnior, pelo apoio incondicional; e, finalmente, aos nossos filhos, que ainda virão... 


\section{Agradecimentos}

À muitíssimo querida Eneida do R. M. Bomfim, pela orientação dedicada, incansável e sábia e pela motivação de todos os dias;

À não menos querida Rosa Marina de B. Meyer, pela coorientação indispensável e pela objetividade nos momentos cruciais;

Aos membros (efetivos e suplentes) da Banca de Tese, pela gentileza em aceitar o meu convite, compreensão com os prazos, leitura cuidadosa e sugestões mais que pertinentes;

À Pontifícia Universidade Católica do Rio de Janeiro, não só pela bolsa de isenção de mensalidades (VRAC), mas também pela oportunidade de crescimento acadêmico;

Aos funcionários do Departamento de Letras da PUC-Rio, em especial à Francisca (Chiquinha), Digerlaine (Di) e Lusinete, pela paciência;

À Assessora para Assuntos Externos da CCCI, Maria Luiza Paranhos (Malu), pela eficiência nas respostas;

À Mônica Maria G. Savedra, membro da Banca de Qualificação, pelas sugestões apropriadas;

Aos professores do Programa de Pós-graduação em Letras da PUC-Rio, pelos preciosos conselhos e ensinamentos;

Aos colegas do doutorado e ex-colegas da especialização (Formação de Professores de Português para Estrangeiros), pelo companheirismo e troca intelectual e, claro, pelos momentos de descontração;

Aos professores/colegas/amigos dos cursos de português para estrangeiros da PUC-Rio (CCE e CCCI) - especialmente aos seus coordenadores, Ricardo Borges Alencar e Adriana Ferreira de Souza Albuquerque -, pela disponibilidade em ajudar com os questionários e as redações;

À Patrícia Sobral e à Augusta Vono, pela coleta de valoroso material em Brown University e na FIU (Florida International University), respectivamente;

Aos alunos que preencheram os questionários, enviaram redações e completaram a atividade-extra, pela boa-vontade;

A todos os autores com quem fiz contato por email, pela atenção e simpatia com que me responderam; 
Aos atuais e antigos chefes do TCE-RJ, Leila S. Dias, Vitória Eugênia de V. Z. Araújo, Mauro Henrique da Silva e Neyde F. Cunha; aos assessores da SSE, Marcia Cristina B. Loyola, Maurício M. do Carmo e Rose Meire F. Corrêa; e aos colegas de CST - particularmente, Paulo Roberto V. de Almeida e Sérgio de C. Baena (meus "braços direito e esquerdo") -, por toda compreensão, interesse e coleguismo, principalmente nas épocas de maior dedicação ao doutorado;

Aos colegas de Copep (COE/ECG/TCE-RJ), em especial aos já doutores, Rosa Maria Chaise, Paula Alexandra C. P. Nazareth e Jean Marcel de F. Novo, pela preocupação e estímulo a cada encontro;

Aos eternos companheiros do Coral do TCE-RJ, pelas demonstrações de carinho;

A Luiz Henrique de A. Pereira, da Biblioteca (BBL/TCE-RJ), pelas dicas valiosas nas referências bibliográficas;

A todos os familiares, parentes e amigos, pelo suporte emocional, vibração positiva e muita curiosidade;

A Leonardo C. Cunha e Ronaldo R. Lanzillotti, pela incalculável ajuda com o inglês e o espanhol;

Aos meus sogros, Fátima C. Silva e Eliésio Siva, por se orgulharem de mim, e aos meus cunhados, Rafaelli, Rodrigo e Rachel, pela torcida e confiança;

À Lúcia, por deixar minha casa sempre em ordem;

E, sobretudo, a Deus, pela força, luz e inspiração dia após dia...

Finalmente, a todos aqueles que, por distração, deixei de citar aqui, mas contribuíram direta ou indiretamente com a realização deste sonho. 


\section{Resumo}

Razuk, Renata de Oliveira; Bomfim, Eneida do Rêgo Monteiro (Orientadora); Meyer, Rosa Marina de Brito (Coorientadora). Do Inglês L1 ao Português L3 passando pelo Espanhol L2: transferências em regência/transitividade verbal, com foco nas preposições. Rio de Janeiro, 2008. 334p. Tese de Doutorado - Departamento de Letras, Pontifícia Universidade Católica do Rio de Janeiro.

Do Inglês L1 ao Português L3 passando pelo Espanhol L2: transferências em regência/transitividade verbal, com foco nas preposições transita por temas pouco explorados - não só pela combinação de línguas adotada, como também pela especificidade do fenômeno analisado e pelo tópico gramatical escolhido -, contribuindo para o desenvolvimento de uma área de pesquisa extremamente recente e promissora: a aquisição de terceira língua. Os estudos em AL3 ainda estão muito tímidos na América Latina, mas prementes no Brasil, por conta da crescente vinda de norte-americanos (IL1) já falantes do espanhol (EL2), querendo aprender o português (PL3). É nesse contexto que se faz relevante estudar como as línguas anteriormente aprendidas influenciam a aquisição de uma terceira língua. Esta tese perpassa os estudos contrastivos entre as três línguas em foco, mormente as transferências entre elas; o relativismo linguístico moderado; a corrente funcionalista; conceitos modernos de transitividade, sobretudo a GV; a Análise de Erros (Análise de Transferências); definições de bilinguismo e multilinguismo; e os fatores de influência em AL3. Tudo isso levando-se em conta que o processo de aquisição de terceira língua é mais complexo do que o de segunda. Utilizando-se alguns instrumentos metodológicos - questionários, redações e atividades elucidativas -, foram selecionados os informantes da pesquisa, diagnosticados os problemas mais comuns causados pelas transferências do inglês (L1) e do espanhol (L2) no português (L3) e confrontados os resultados com a hipótese formulada. Verificou-se que quanto maior o domínio na língua espanhola, maior também a sua influência no português, ao mesmo tempo em que diminuem as influências do inglês e aumentam as neutralizações das suas interferências. Contudo, não se pode dizer que a competência no espanhol é determinante para que a L2 seja mais utilizada que a L1 como base de transferências, vez que os índices de incidência do inglês continuam altos mesmo nos alunos com maior domínio daquela língua. Apesar de o espanhol ter alguns fatores a seu favor: maior proximidade tipológica com a L3, o status de LE e o efeito de última língua aprendida; vale destacar essa prevalência da LM, que talvez seja decorrente da escolha da sintaxe como objeto de estudo. Outro objetivo alcançado com a pesquisa foi a formulação de matrizes de transferências que podem auxiliar o professor em sala de aula de PLE. Ciente das diferenças entre as regências, ele é capaz de propor atividades específicas para ajudar esse grupo de alunos a empregar as preposições adequadamente no português, sem incentivá-los a fazer traduções literais - vício bastante encontrado nas gramáticas de português voltadas para o estrangeiro. $O$ presente estudo ainda sugere temas para pesquisas futuras, como, por exemplo, a utilização de outra constelação de línguas ou a mudança do foco da análise mantendo o mesmo perfil de alunos. Pelo ineditismo do tema e dos resultados obtidos, esta tese pretende, em última instância, enriquecer o meio acadêmico colaborando com a descrição do português, não somente como língua estrangeira, mas principalmente como terceira língua.

\section{Palavras-chave}

Aquisição de terceira língua (AL3); línguas em contato; inglêsL1-espanholL2portuguêsL3; análise de transferências; regência/transitividade verbal; preposições; gramática de valências; relativismo linguístico; ensino de português como língua estrangeira; aplicações pedagógicas. 


\section{Abstract}

Razuk, Renata de Oliveira; Bomfim, Eneida do Rêgo Monteiro (Supervisor); Meyer, Rosa Marina de Brito (Co-Supervisor). From English as L1 to Portuguese as L3 through Spanish as L2: transfers in verb regency/transitivity, with special emphasis on prepositions. Rio de Janeiro, 2008. 334p. Doctoral (PhD) Thesis - Departamento de Letras, Pontifícia Universidade Católica do Rio de Janeiro.

From English as L1 to Portuguese as L3 through Spanish as L2: transfers in verb regency/transitivity, with special emphasis on prepositions handles topics that have not yet been deeply explored. In addition to a unique combination of languages, this thesis focus on a specific phenomenon and grammar item, thus providing an important contribution for the development of an extremely recent (and promising) research area Third Language Acquisition. Although TLA studies are incipient in Latin America, in Brazil one may see the urge to adapt a new linguistic reality as more and more Spanishspeaking North Americans who have come to the country want to learn Portuguese. That would call for some investigation on the influences between previous learned languages and the target one. Theory includes contrastive studies, particularly transferences among the three tongues selected; moderate linguistic relativism; the functionalist school; modern transitivity concepts, especially Valence Grammar; Error Analysis (Transference Analysis); bilingualism / multilingualism definitions; and TLA influence factors. An underlying consideration is the fact that the acquisition of a third language is more complex than the process to acquire a second language. By means of some tools (questionnaires, compositions and elucidative activities), research reporters were selected; the most common errors caused by English (L1) / Spanish (L2) transferences into Portuguese (L3) were diagnosed; and the results were then compared against the proposed hypothesis. Data analysis indicated that the better expertise one has in Spanish, the higher is their Portuguese influence as the amount of $\mathrm{L} 2$ transference in $\mathrm{L} 3$ rises along Subgroups (C - low; B - average; A - high). Meanwhile, English influence in Portuguese performance decreases and interference neutralizing rises. It would not be possible to affirm, however, that Spanish command is essential for L2 to be more frequently employed than L1 as a basis for transference, given that English indices are still high in the 'best command' subgroup. Despite Spanish 'pros' - higher typology proximity to TL; FL status; and the last language effect -, one's MT prevalence may result from verb transitivity choice as an object of study. Another target that has been achieved by this thesis is the formulation of transference matrices that may help Portuguese as Foreign Language teachers in their classrooms. Once they are aware of agreement differences, they will be able to come up with specific activities to help these students use Portuguese prepositions correctly, instead of encouraging them to transfer directly from one language into another - unfortunately, most PFL grammars does so. For the future, it would be interesting, for example, to develop a similar study with other constellations of languages or to choose another object while keeping the profile selected in here. By and large, this work intends to enrich the academia by collaborating to describe Portuguese as a third language (rather than just a 'foreign language'), as pioneer handling of topics and the results that have been achieved should qualify this research for future reference.

\section{Keywords}

Third language acquisition (TLA); languages in contact; EnglishL1-SpanishL2PortugueseL3; transfer analysis; verb regency/transitivity; prepositions; valence grammar; linguistic relativity; teaching of portuguese as a foreign language; pedagogical practices. 


\section{Sumário}

$\begin{array}{ll}\text { Apresentação breve } & 14\end{array}$

1. Introdução 15

1.1. O tema e suas delimitações $\quad 15$

1.1.1. Trabalhos anteriores/motivadores $\quad 15$

$\begin{array}{ll}\text { 1.1.2. O problema atual } & 17\end{array}$

$\begin{array}{ll}\text { 1.2. Justificativas teóricas e práticas } & 18\end{array}$

1.3. Relevância acadêmico-pedagógica 23

1.4. Hipótese $\quad 24$

1.5. Objetivos 26

2. Revisão da literatura 28

2.1. Sobre o contato entre as línguas e suas consequências 28

2.1.1. Contrastes inglês-português 28

2.1.2. Contrastes espanhol-português 30

2.1.3. Contrastes inglês-espanhol 34

2.2. Sobre a aquisição de terceiras línguas (AL3) 35

2.2.1. Aspectos gerais, fatores de influência e alguns exemplos 36

2.2.2. Bilinguismo e trilinguismo 42

2.2.3. A sintaxe em L3 45

2.2.4. Inglês, espanhol e português 51

3. Fundamentação teórica 54

3.1. "Pano de fundo": correntes teóricas 54

3.1.1. Universalismo ou relativismo 54

3.1.2. O funcionalismo e a gramática de valências 57

3.1.3. Regência/transitividade verbal: tradição e modernidade 61

3.2. Linguística aplicada ao ensino de línguas estrangeiras 67

3.2.1. Linguística contrastiva, análise de erros e interlíngua 67

3.2.2. Análise de transferências $\quad 71$

3.2.3. A transferência como estratégia, as LNNs como fonte e a GU 76

3.3. Aquisição de terceira língua 82

3.3.1. O estado da arte $\quad 82$

3.3.2. Fatores de influência 86

3.3.3. Aquisição multilíngue $\quad 91$

3.4. As línguas em contato 95

3.4.1. Definições de outros termos relevantes 95

3.4.2. Inglês, espanhol e português: comparação das estruturas 101

$\begin{array}{ll}\text { 3.4.2.1. Línguas próximas } & 101\end{array}$

$\begin{array}{ll}\text { 3.4.2.2. Línguas distantes } & 108\end{array}$

4. As gramáticas do português como língua estrangeira 115 
5. Procedimentos metodológicos 123

5.1. Tipo de pesquisa 123

5.2. Método: seleção dos sujeitos e aplicação dos instrumentos 124

5.2.1. O questionário 125

5.2.2. As redações 127

5.2.3. Atividade Complementar (teste) 129

5.3. Limitações do método 129

6. Análise dos dados 133

6.1. Os questionários 133

6.2. As redações: Grupo 1 (IL1 e PL2) e Grupo 2 (EL1 e PL2) 135

6.2.1. Grupo 1: inglês L1 e português L2 136

6.2.2. Grupo 2: espanhol L1 e português L2 146

6.2.3. Matrizes dos Grupos 1 e 2: padrões de transferências 151

6.3. As redações: Grupo 3 (IL1, EL2 e PL3) 152

6.3.1. Subgrupo C: IL1, EL2 (domínio baixo) e PL3 154

6.3.2. Subgrupo B: IL1, EL2 (domínio médio) e PL3 155

6.3.3. Subgrupo A: IL1, EL2 (domínio alto) e PL3 156

6.3.4. Interpretação dos resultados 156

6.4. A atividade complementar (teste) 158

6.5. Resultados comparativos: redações e atividades 158

$\begin{array}{ll}\text { 7. Aplicações pedagógicas } & 167\end{array}$

8. Conclusões e considerações finais 170

9. Referências bibliográficas 175

Anexo 1: Censo Populacional de 2000 - EUA 191

Anexo 2: Censo Populacional de 2005 (prévia para 2010) - EUA 196

Anexo 3: Questionários (versões PUC-Rio, Brown e FIU) 198

Anexo 4: Atividade complementar (teste) 202

Anexo 5: transferências do Grupo 1 (IL1 e PL2) 207

Anexo 6: transferências do Grupo 2 (EL1 e PL2) 216

Anexo 7: transferências do Grupo 3 - Subgrupo C 223

Anexo 8: transferências do Grupo 3 - Subgrupo B 235

Anexo 9: transferências do Grupo 3 - Subgrupo A 247

Anexos 10-21: Respostas à atividade complementar - Aluno $\mathrm{X}$, Subgrupo $Y \quad 257$

Anexo 22: Compilação dos resultados da atividade complementar 324 


\section{Lista de siglas}

LE: língua estrangeira (ou $F L$ : foreign language) $=$ LNN: língua não-nativa = LNM: língua não-materna

L1: primeira língua $=\mathrm{LN}$ : língua nativa (ou NL: native language) $=\mathrm{LM}$ : língua-mãe/materna (ou MT: mother tongue)

L2: segunda língua = primeira LE/LNN/LNM

L3: terceira língua = segunda LE/LNN/LNM

L4: quarta língua = terceira LE/LNN/LNM

L5: quinta língua = quarta LE/LNN/LNM

LA: língua-alvo/meta (ou TL: target language) X LF: língua-fonte/de origem (ou source language)

PLE: português como língua estrangeira = PLNN: português como língua não-nativa = PLNM: português como língua não-materna

PL1: português como primeira língua $=$ PLN: português como língua nativa $=$

PLM: português como língua-mãe/materna

PL2: português como segunda língua ou primeira LE/LNN/LNM

PL3: português como terceira língua ou segunda LE/LNN/LNM

EL1: espanhol como primeira língua = ELN: espanhol como língua nativa $=$

ELM: espanhol como língua-mãe/materna

EL2: espanhol como segunda língua ou primeira LE/LNN/LNM

IL1: inglês como primeira língua = ILN: inglês como língua nativa = ILM: inglês como língua-mãe/materna

IL2: inglês como segunda língua ou primeira LE/LNN/LNM

ALE: aquisição de língua estrangeira

AL1: aquisição de primeira língua

AL2: aquisição de segunda língua (ou SLA: second language acquisition)

AL3: aquisição de terceira língua (ou TLA: third language acquisition) 
AML: aquisição multilíngue (ou MLA: multilingual acquisition)

IL: interlíngua (ou IL: interlanguage)

CLI: influências interlinguísticas (ou CLI: cross-linguistic influences) ${ }^{1}$

GV: Gramática de Valências

RV: regência verbal

GU: Gramática Universal (ou UG: Universal Grammar)

${ }^{1}$ A sigla em português foi mantida como no inglês para seguir a literatura dominante. 
"(...) Eu me sinto um estrangeiro, passageiro de algum trem, que não passa por aqui, que não passa de ilusão (...)" (música: Revolta dos Dândis I - por Engenheiros do Havaí)

"(...) I feel like a foreigner, a passenger in a train, that doesn't pass here, that means nothing but illusion (...)" (song: The Dandies' Rebellion - by The Engineers from Hawaii)

“(...) Me siento como si fuera un extranjero, pasajero de algún tren, que no pasa por acá, que no pasa de una ilusión (...)" (melodía: La Sublevación de los Presumidos - de Los Ingenieros de Havaí) 


\section{Apresentação breve ${ }^{2}$}

Este trabalho expõe os resultados de uma pesquisa de tese (investigação de caráter original para fins de doutoramento), cujo tema-central está claramente indicado em seu título: Do Inglês L1 ao Português L3 passando pelo Espanhol L2: transferências em regência/transitividade verbal, com foco nas preposições. O texto se organiza nas seguintes partes: Cap. 1 - Introdução; Cap. 2 - Revisão da literatura; Cap. 3 - Fundamentação teórica; Cap. 4. As gramáticas do português como língua estrangeira; Cap. 5 - Procedimentos metodológicos; Cap. 6 - Análise dos dados; Cap. 7 - Aplicações pedagógicas; Cap. 8 - Conclusões e considerações finais; e Cap. 9 - Referências bibliográficas. A exceção dos dois últimos capítulos, por desnecessário, todos receberam um resumo para facilitar a compreensão do leitor. Esta tese conta ainda com uma Lista de siglas, logo após o Sumário, e Anexos (01-22), ao final.

\footnotetext{
${ }^{2}$ A elaboração do texto e a formatação da tese seguem o conjunto de procedimentos apresentados pelo manual Pós-Graduação PUC-Rio: normas para apresentação de teses e dissertações (Pontifícia Universidade Católica do Rio de Janeiro, 2001). Além disso, já foram adotadas aqui as alterações introduzidas na ortografia do português pelo Acordo Ortográfico da Língua Portuguesa, assinado em Lisboa em 16 de dezembro de 1990 e aprovado no Brasil pelo Decreto Legislativo $\mathrm{n}^{\circ}$ 54, de 18 de abril de 1995 - exceto nas referências, citações e anexos, que foram mantidos propositalmente como nos seus originais. Observe-se que a implantação dessas regras no nosso país está prevista para ocorrer a partir de 2009 - quando esta tese passará a estar disponível na Biblioteca da PUC-Rio -, mas com um período de transição até 2012. Portanto, apesar de obrigatória somente de 01 de janeiro de 2013 em diante, a adoção imediata das novas normas certamente dará maior "longevidade ortográfica" a esta pesquisa.
} 